\title{
Revisión sistemática: Formación continua de docentes en las plataformas virtuales
}

\author{
Systematic review: In-service teacher training on virtual platforms
}

\author{
Sujeidy María Cotohuanca Cruz ${ }^{1}$ \\ ${ }^{1}$ Universidad Cesar Vallejo
}

\begin{abstract}
Resumen
Las deficiencias detectadas en los docentes con respecto al uso de las plataformas virtuales resultan un problema relevante dada la importancia del momento actual, en donde las clases presenciales pasaron a ser virtuales debido a la pandemia mundial. En ese sentido, el objetivo del estudio es sistematizar los estudios científicos referentes a la formación continua de docentes en las plataformas virtuales entre los años 2016 y 2020, en las bases de datos de Ebsco y Scielo. La metodología empleada fue una revisión exhaustiva de artículos, utilizando términos como: formación continua, docentes y plataformas virtuales. Los criterios incluidos fueron: fuente, población-muestra y objetivo. Los resultados evidenciaron siete artículos sobre formación continua de docentes. Los principales resultados son: las limitaciones que tienen los docentes en el uso de las plataformas virtuales y las orientaciones que reciben para mejorar el aprendizaje en la educación universitaria. En conclusión, es necesario (1) desarrollar programas de capacitación en el uso de plataformas virtuales para el logro de competencias del aprendizaje en estudiantes de educación universitaria y (2) orientar nuevas investigaciones centradas en la formación continua de profesores en plataformas virtuales.
\end{abstract}

Palabras clave: formación continua; docentes; plataforma virtual; educación.

\begin{abstract}
The deficiencies detected in teachers regarding the use of virtual platforms is a relevant problem given the importance of the current moment, where face-to-face classes became virtual due to the global pandemic. In that sense, the objective of this study is to systematize the scientific studies referring to the continuous training of teachers in virtual platforms between the years 2016 and 2020, in the EBSCO and SCIELO databases. The methodology used was an exhaustive review of articles, using terms such as: continuous training, teachers and virtual platforms. The criteria included were:
\end{abstract}


source, population-sample and objective. The results showed seven articles on continuing teacher training. The main results are: the limitations that teachers have in the use of virtual platforms and the orientations they receive to improve university education learning. In conclusion, it is necessary (1) to develop training programs in the use of virtual platforms for the achievement of learning competences in university education students and (2) to guide new research focused on the continuous training of teachers in virtual platforms.

Keywords: continuous training; teacher; virtual platform; education.

\section{Introducción}

La ciencia está avanzado a grandes pasos en el desarrollo de la educación. En ese sentido, la tecnología ha experimentado un crecimiento acelerado y las instituciones educativas están incorporando los medios tecnológicos al aprendizaje con la aplicación de diversas plataformas virtuales que llevan a los docentes a nuevos cambios en la didáctica y metodología. Al respecto, Sánchez (2019) indicó que el nuevo paradigma de aprendizaje está evolucionando dentro de la formación virtual con la utilización de herramientas que permitan la comunicación y den un rol más activo al estudiante. Asimismo, Muñoz y Vargas (2019) señalan un método de enseñanza de desarrollo en la aplicación web con un ambiente interactivo, participativo y didáctico donde interviene el beneficio de insertar las habilidades y estrategias didácticas de aprendizaje en las aulas. También, Mejía (2018) da a conocer la importancia que genera el impacto de la herramienta web Edmodo en los estudiantes, así como la calidad de la gestión de prácticas escolares planificadas en la plataforma. En el mismo sentido, Cabero (2018) manifiesta diferentes propuestas que permitan identificar los elementos y estrate- gias en un modelo evaluativo aplicando plataformas virtuales que permitan mejorar su actividad profesional como parte de los procesos virtuales en la evaluación del desempeño docente.

Valencia y Rodríguez (2018) manifies$\tan$ que las herramientas de la Web 2.0 contribuyen a un aprendizaje colaborativo para demostrar sus habilidades en los estudiantes. En el mismo sentido, Gómez y Muriel (2018) expresan que los docentes buscan desarrollar las habilidades y destrezas de sus estudiantes al usar las plataformas digitales para facilitar un aprendizaje significativo y útil con el cual se pretende afianzar el proceso de enseñanza aprendizaje según su contexto.

Pérez y Hernández (2018) exponen las consecuencias de un programa fundamentado en competencias digitales aplicado en metodologías didácticas de affective elearning en la comprensión y aplicación de habilidades de la competencia digital. Asimismo, Unesco (2018) señala que los maestros protagonizan uno de los poderes más sólidos y de gran influencia con la expectativa de garantía de la igualdad, la accesibilidad y la calidad de la educación. Estos son la esencia del progreso del mundo sostenible.

Srnicek (2017) indica que las platafor- 
mas son herramientas digitales que acceden a que dos o más agrupaciones interactúen. Por consiguiente, se sitúan como mediadores que congregan a diversos usuarios: clientes, anunciadores, vendedores de servicios, fabricantes, proveedores e incluso objetos físicos. Asimismo, Jofré (2009) señala que el desempeño docente se estima por medio de la dinámica de recursos intelectuales profesionales para usar definidos objetos de aprendizaje mediante metodologías didácticas oportunas con profesionalismo ético en un escenario determinado.

En estos tiempos las plataformas virtuales involucran la aplicación de tecnología en la labor docente garantizando mejorar la calidad y equidad educativa en ambientes de aprendizaje online. De ese modo, permiten al docente desarrollar todos los contenidos, métodos, técnicas y estrategias que van a permitir interactuar mejor en el proceso de enseñanza - aprendizaje con el alumno, tomando en cuenta los diferentes contextos, necesidades e intereses de cada uno de ellos.

Las plataformas virtuales son portales que permiten interactuar y/o almacenar diferentes tipos de información, permita además acceder a la misma en cualquier momento y desde cualquier lugar, lo que facilita el trabajo de manera más efectiva y productiva, al utilizar herramientas para gestionar sus actividades de manera más sencilla para los usuarios.

Por lo expuesto, la pregunta de investigación que guía el estudio es: ¿Cómo se evidencia en los artículos la formación continua de docentes en las plataformas virtuales? Y el objetivo planteado es analizar la información en la formación con- tinua de docentes en las plataformas virtuales que favorecen a perfeccionar el procedimiento de enseñanza - aprendizaje del docente.

\section{Metodología}

Se constituyó en un estudio sistemático de artículos indizados en las bases de datos Ebsco, y Scielo dentro del marco académico y científico de las ciencias educativas de los años 2016-2020. En el desarrollo del método se formuló la pregunta, planificó los criterios de elegibilidad, buscó los estudios, aplicó los criterios de elegibilidad, se obtuvo los datos, evaluó el riesgo de sesgo de los estudios, analizó y presentó los resultados. Se aplicó el método prisma.

Proceso de selección de los artículos

En la revisión sistemática se realizó el siguiente procedimiento, los criterios de elegibilidad de los artículos tenían las siguientes características: 1) publicaciones de tipo teóricas o empíricas que aportaran antecedentes a la formación continua de docentes en plataformas virtuales; 2) los participantes son estudiantes universitarios y 3) el idioma de búsqueda fue el el español. En este apartado se describe paso a paso el desarrollo de la revisión sistemática de las plataformas virtuales Ebsco y Scielo, siguiendo un orden de búsqueda específica, los cuales se observan en la Tabla 1. 


\begin{tabular}{|l|c|l|}
\hline Refinación de búsqueda & \multicolumn{1}{|c|}{ Ebsco } & \multicolumn{2}{|c|}{ Scielo } \\
\hline Nombre de los descriptores & $\begin{array}{l}\text { Formación continua, docentes y } \\
\text { plataformas virtuales. }\end{array}$ & $\begin{array}{l}\text { Formación } \\
\text { docentes y plataformas } \\
\text { virtuales. }\end{array}$ \\
\hline $\begin{array}{l}\text { Ubicación de descriptores } \\
\text { "and", "or" }\end{array}$ & \multicolumn{2}{|c|}{ En el tema } \\
\hline Periodo de tiempo & \multicolumn{2}{|c|}{ Educación } \\
\hline Áreas de investigación & \multicolumn{2}{|c|}{ Articulo } \\
\hline Tipo de documento & \multicolumn{2}{|c|}{ Español } \\
\hline Idioma & $\begin{array}{l}\text { 52 articulos encontrados. } \\
\text { Cumplen con el criterio de } \\
\text { inclusión de 6 articulos. }\end{array}$ & $\begin{array}{l}\text { Trece articulos encontrados. } \\
\text { Cumplen con el criterio de } \\
\text { inclusión de 1 articulo. }\end{array}$ \\
\hline Resultados de búsqueda
\end{tabular}

Tabla 1: Criterios específicos de elegibilidad para la búsqueda sistemática de artículos

\section{Resultados de la búsqueda (dia- grama de flujo - Prisma)}

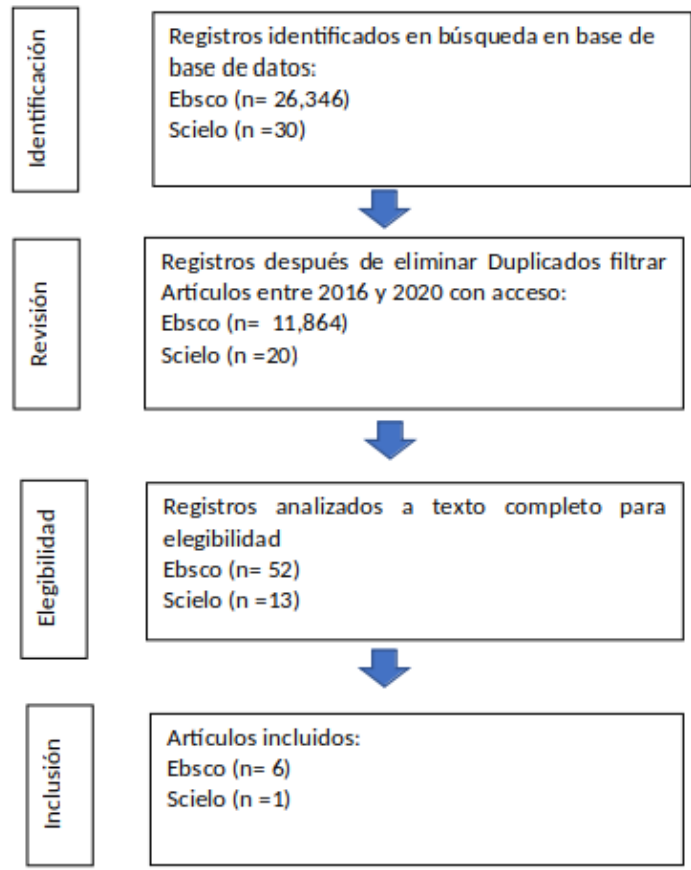

Análisis de características de los

\section{artículos seleccionados}

En el registro de datos de los artículos investigados, se organizó la información basado en los siguientes campos: Fuente, Muestra Población y Objetivo (Tabla 2).

Resultados de la revisión de los artículos seleccionados

Se analizaron artículos en idioma español, y se hallaron siete investigaciones latinoamericanas que satisfacen las pautas de inclusión. Las muestras fluctúan desde 19 a 251 alumnos.

Los artículos están relacionados con los siguientes objetivos a la aplicabilidad de plataformas virtuales en la labor docente. Primordialmente se plantean objetivos respecto a tres temas: (1) aplicación de plataformas para complementar las acciones educativas, (2) interacción con las plataformas virtuales en sesiones sincrónicas y asincrónicas, (3) empleo de herramientas digitales y su aplicabilidad en las sesiones de enseñanza-aprendizaje.

\section{Criterios de inclusión}




\begin{tabular}{|l|l|c|l|}
\hline \multicolumn{1}{|c|}{$\mathbf{N}^{\circ}$} & \multicolumn{1}{|c|}{ Fuente } & $\begin{array}{c}\text { Tamaño de } \\
\text { muestra }\end{array}$ & \multicolumn{1}{c|}{ Objetivo } \\
\hline 1. & $\begin{array}{l}\text { Vilora Matheus, Hender Alexander } \\
\text { Hamburger, Javier (2019) }\end{array}$ & 87 & $\begin{array}{l}\text { "Mejorar el manejo de herramientas comunicativas en entornos virtuales de aprendizaje y } \\
\text { generar aportes innovadores en el ámbito educativo". }\end{array}$ \\
\hline 2. & $\begin{array}{l}\text { Vázquez Cano, Esteban } \\
\text { López Meneses, Eloy } \\
\text { Fernández Márquez, Esther } \\
\text { Ballesteros Regaña, Cristóbal (2018) }\end{array}$ & 251 & $\begin{array}{l}\text { "Investigar la percepción relativa a las ventajas de los cursos MOOC en los ámbitos socio- } \\
\text { educativos que emplean el estudiantado del primer curso de la asignatura de Tecnologías de } \\
\text { la Información y la Comunicación (TIC)". }\end{array}$ \\
\hline 3. & Mejía Salazar, Gilberto (2018) & 81 & $\begin{array}{l}\text { "Conocer el impacto que genera la plataforma educativa EDMODO en los estudiantes, así } \\
\text { como la importancia de la gestión de actividades escolares dentro de dicha plataforma". }\end{array}$ \\
\hline 4. & $\begin{array}{l}\text { Valencia Vivas Gloria Maritza } \\
\text { Rodríguez Rafael Glen Dario (2018) }\end{array}$ & 100 & $\begin{array}{l}\text { "Identificar la contribución efectiva de las herramientas de la Web 2.0 en el rendimiento } \\
\text { académico a través del aprendizaje colaborativo para contribuir a la eficiencia y la eficacia } \\
\text { de la educación". }\end{array}$ \\
\hline 5. & $\begin{array}{l}\text { Muñoz Sanabria, Luis F. } \\
\text { Vargas Ordoñez, Leydi M. (2019) }\end{array}$ & 19 & $\begin{array}{l}\text { "Promover las mejores prácticas en el contexto de la educación a través de la creación de } \\
\text { una herramienta web para la enseñanza de las operaciones elementales, que permita } \\
\text { mejorar el desempeño de los estudiantes de básica primaria en matemáticas". }\end{array}$ \\
\hline 6. & $\begin{array}{l}\text { Ocampo López, Arturo } \\
\text { Gómez Zermeño, Marcela Georgina } \\
\text { Zambrano Izquierdo, David (2016) }\end{array}$ & 30 & $\begin{array}{l}\text { "Mostrar la aceptación de los participantes del uso de la plataforma educativa Blackboard, } \\
\text { con el refuerzo de sesiones de asesorías presenciales que les permiten prepararse para } \\
\text { intervenir en procesos de certificación". }\end{array}$ \\
\hline 7. & $\begin{array}{l}\text { Gómez López, Luis Felipe } \\
\text { Silas Casillas, Juan Carlos (2016) }\end{array}$ & 30 & $\begin{array}{l}\text { "Mejorar la práctica docente en la aplicación de la plataforma Moodle en el área de } \\
\text { matemática y su interacción con las herramientas brindadas". }\end{array}$ \\
\hline
\end{tabular}

Tabla 2: Análisis de las características de los artículos sobre aplicación de plataformas virtuales.

Fuente: Elaboración propia.

Durante la búsqueda, se tuvo en cuenta las publicaciones que trabajen con plataforma virtual o digital, que mostraran la evidencia científica sobre formación continua de docentes, sin interesar el modelo teórico y las técnicas aplicadas. También, se incorporaron exclusivamente investigaciones de carácter cuantitativo y/o con un modelo experimental o mixto que fuera explícitos en las técnicas y pruebas aplicadas, dentro del rango de tiempo comprendido entre el 2016 y 2020.

\section{Criterios de exclusión}

No se tendrían en cuenta estudios cualitativos. También se excluyeron revisiones bibliográficas, análisis de casos, revisiones sistemáticas.

Resultados del proceso de análisis de los artículos en formación continua de los docentes en plataformas virtuales

1. "Las herramientas comunicativas en el ámbito virtual de aprendizaje". (Vilora y Hamburger, 2019). Se refiere al uso de medios tecnológicos para una comunicación sincrónica y asincrónica en el ámbito virtual de aprendizaje. Se realizó el análisis con una muestra de 87 alumnos y docentes. Se concluyó que los docentes aprovechan mayormente algunos medios digitales como correo electrónico y la pizarra electrónica, entretanto otras herramientas se usan a medias por desconocimiento. Por esto, se proponen crear estructuras de planificación para los docentes en su mejora de utilización de herramientas comunicativas en ámbitos virtuales de aprendizaje y forjar contribuciones innovadoras en el campo educacional.

2. "Los nuevos ámbitos virtuales de aprendizaje continuo (MOOC) y sus 
posibilidades educativas en ámbitos sociales y educativos". (Vázquez, López, Fernández y Ballesteros, 2018). Se refiere al estudio sincrónico de las fortalezas de los estudiantes a través del curso MOOC. La muestra está dada por 251 estudiantes que aplicaron metodologías descriptiva y cualitativa, las consecuencias más sobresalientes señalan las herramientas que proporciona en entorno virtual MOOC, también ayuda a los docentes que tienen menos habilidades con las herramientas tecnológicas para compartir opiniones y prácticas educativas y contenidos universitarios.

3. "Uso de la plataforma educativa Edmodo como recurso para la gestión de actividades escolares". (Mejía, 2018). Consistió en conocer la utilidad de herramientas que presenta la plataforma educativa Edmodo en los estudiantes. Se llevaron a cabo actividades diversas, aplicando las múltiples opciones que nos brinda la plataforma, tales como la consulta de actividades como textos, videos, sonidos, tareas, etc., así como el envío de trabajos y proyectos académicos; de ese modo, los docentes van a tener una herramienta que mejora considerablemente la atención de los estudiantes. Cabe mencionar, que Edmodo es una plataforma muy interactiva con interfaces intuitivas y presenta una comunicación segura, clara, fluida y constante como cualquier proyecto. Por lo tanto, con la plataforma se va a promover el interés y creatividad por los alumnos en aplicar hoy en día las tecnologías en su labor educativa.

4. "Revisión bibliográfica de estudios empíricos de herramientas de la Web 2.0 para el aprendizaje colaborativo: wikis, blogs, redes sociales y repositorios multimedia". (Valencia y Rodríguez, 2018). Consistió en un aprendizaje colaborativo que involucra aplicar herramientas Web 2.0 en el campo educativo, con el fin de contribuir en el rendimiento académico por medio de estas herramientas que tienen como fin la aplicación y coadyuvar al fortalecimiento de la eficiencia y eficacia en bien de la educación. Se incluyó que las herramientas Web 2.0 van a permitir el aprendizaje colaborativo en los estudiantes, promoviendo su participación en todos los procesos de enseñanza - aprendizaje.

5. "Edumat: herramienta web gamificada para la enseñanza de operaciones elementales". (Muñoz y Vargas, 2019). Consistió en la aplicación de un método muy utilizado en la enseñanza de operaciones elementales que están fundamentadas en las TIC. Consiste la aplicación web en un ambiente interactivo y didáctico; mediante juegos, se probó las habilidades y destrezas que adquirían los alumnos. El propósito fue comprobar por medio de una prueba de implantación en un ámbito educativo si el método es verdaderamente efectivo y si provoca la motivación por hacer que formen parte las estrategias 
didácticas de aprendizaje en los ambientes de clase.

6. "Percepción del profesor sobre el uso del b-learning para fortalecer competencias laborales". (Ocampo y Gómez, 2016). Consistió en la aplicación de un aprendizaje combinado llamado blended learning que desarrolla competencias en los facilitadores en la utilización básica de los tics. Se aplicó cuestionarios y entrevistas para incorporar proyectos de aprendizaje en los contenidos y recursos. Los resultados arrojan el acercamiento de los partícipes en el uso de la plataforma educativa Blackboard, con la ayuda de acompañamientos de asesoría presencial que les permite obtener preparación para participar en distintos procedimientos de certificación.

7. "La comunidad virtual de práctica. Alternativa para la formación continua de profesores" (Gómez y Silas, 2016), Consistió en la aplicación de una comunidad virtual con la plataforma informática Moodle, que tiene como prioridad el mejoramiento del quehacer docente de los responsables del área de matemática que instruyen en el primer grado de secundaria. En la plataforma subían su información semanal interactuando con las herramientas que proporciona Moodle, a través de 35 foros semanales durante el año lectivo escolar. El método de análisis empleado fue cualitativo y da cuenta, en primer lugar, de la creación de comunidades de aprendizaje; y en segundo lugar, de la valoración que llevan a cabo tomando en cuenta su intervención en esta modalidad de formación continua.

\section{Discusión}

La presente investigación tiene como objetivo analizar la información en formación continua de docentes en las plataformas virtuales que favorecen a perfeccionar el procedimiento de enseñanza - aprendizaje del docente. Coincide con los resultados de otros estudios que resaltan la necesidad de implementación de estos programas. Al respecto, Cabero (2018) manifiesta diferentes propuestas que permitan identificar los elementos y estrategias en un modelo evaluativo aplicando plataformas virtuales con vistas a mejorar su actividad profesional como parte de los procesos virtuales en la evaluación del desempeño docente. En los artículos revisados, según Ocampo - Gómez, GómezSilas, 2016, estas plataformas virtuales ayudan a desarrollar las competencias laborales que permite mejorar la práctica docente en la interacción con las herramientas digitales y reforzando las sesiones de aprendizaje.

También, Mejía (2018) da a conocer la importancia que genera el impacto de la herramienta web Edmodo en los estudiantes, así como la calidad de la gestión de prácticas escolares planificadas en la plataforma. Al respecto, Vázquez et. Al. y Mejía (2018) aplicaron entornos virtuales en sus sesiones para incrementar mejor sus ideas y expectativas en su labor docente generando una mayor interacción y comunicación con sus alumnos y la plataforma, 
empleando sus opciones de interfaz que proporciona y promoviendo el interés de utilizar estas herramientas tecnológicas.

También Vilora et. al. (2019) y Valencia - Rodríguez (2018) interactuaron con las múltiples aplicaciones de herramientas que nos brinda la Web 2.0, aprovechando sus variadas opciones. Por ello, proponen crear métodos de una continua formación a los maestros con el fin de mejorar la utilización y aplicabilidad de las herramientas digitales de aprendizaje y poder generar grandes aportes innovadores en el campo educativo. En el mismo sentido, Valencia y Rodríguez (2018) manifiestan que las herramientas de la Web 2.0 contribuyen a un aprendizaje colaborativo para demostrar sus habilidades en los estudiantes.

Asimismo, Gómez y Muriel (2018) expresan que los docentes buscan en los estudiantes desarrollar sus habilidades y destrezas al usar las plataformas digitales para facilitar un aprendizaje significativo y útil con la cual se pretende afianzar el proceso de enseñanza y aprendizaje según su contexto. Asimismo, Pérez y Hernández (2018) exponen las consecuencias de un programa fundamentado en competencias digitales aplicado en metodologías didácticas de affective e-learning en la comprensión y aplicación de habilidades de la competencia digital. Asimismo, Unesco (2018) señala que los maestros protagonizan uno de los poderes más sólidos y de gran influencia con la expectativa de garantizar la igualdad, la accesibilidad y la calidad de la educación. Estos son la esencia del progreso del mundo sostenible.

Srnicek (2017) indica que las plataformas son herramientas digitales, y acce- den a que un par o más agrupaciones interactúen. Por consiguiente, se sitúan como mediadores que congregan a diversos usuarios: clientes, anunciadores, vendedores de servicios, fabricantes, proveedores e incluso objetos físicos. Asimismo, Castro (2015), señala que el desempeño docente se estima por medio de la dinámica de recursos intelectuales profesionales para usar definidos objetos de aprendizaje mediante metodologías didácticas oportunas con profesionalismo ético en un escenario determinado.

\section{Conclusiones}

Se concluye que la formación continua de docentes en plataformas virtuales ayuda al proceso de enseñanza - aprendizaje, al aplicar las nuevas tecnologías para mejorar la labor del profesor en beneficio del estudiante apoyándolo en su formación integral. Los docentes deben capacitarse mediante programas que contengan las múltiples herramientas tecnológicas, para proveerse de recursos educativos virtuales.

\section{Referencias}

(1) Arancibia M., Cabero J. y Marín V. (2020). Creencias sobre la enseñanza y uso de las tecnologías de la información y la comunicación (TIC) en docentes de educación superior. Revista Scielo, Formación universitaria versión On-line ISSN 0718-5006, Form.Univ. vol.13 no.3. La Serena jun. 2020 http: 
//dx.doi.org/10.4067/S0718$50062020000300089 \backslash$ protect $\backslash$ unhbox \voidb@x \penalty $@ M \backslash$

(2) Barrera V. y Guapi A. (2018). La importancia del uso de las plataformas virtuales en la educación superior, Revista Atlante: Cuadernos de Educación y Desarrollo (julio 2018). En línea: https://www.eumed.net/rev/ atlante/2018/07/plataformasvirtuales-educacion.html/ /hdl.handle.net/20.500.11763/ atlante1807plataformas- virtualeseducacion

(3) Canales, A., Fernández, M. y Ulate, G. (2020). Aprender y enseñar con recursos TIC: experiencias innovadoras en la formación docente universitaria. Revista Ensayos Pedagógicos, 15(1), 235-248. https:// doi.org/10.15359/rep.15-1.12

(4) Cabero, J., Llorente, M ${ }^{\mathrm{a}}$., Morales, J. (2018). Evaluación del desempeño docente en la formación virtual: ideas para la configuración de un modelo RIED. Revista Iberoamericana de Educación a Distancia, vol. 21, núm. 1, 2018 Asociación Iberoamericana de Educación Superior a Distancia, Brasil Disponible en: http://www.redalyc.org/articulo. oa?id=331455825014 DOI: https: //doi.org/10.5944/ried.21.1.17206

(5) García, A. (2015) Frecuencia de uso de las plataformas virtuales de enseñanza. Una comparación
Moodle versus Sakai en los estudios de perfil económico. Revista de Investigación en Educación, [S.l.], v. 13, n. 1, p. 69-87, abr. 2015. ISSN 2172-3427. Disponible en: http://reined.webs.uvigo.es/ index.php/reined/article/view/242

(6) Gómez, L y Silas, J. (2016). La comunidad virtual de práctica. Alternativa para la formación continua de profesores. ISSN 1870-5308

(7) Hernández C. y Tecpan S. (2017). Aula invertida mediada por el uso de plataformas virtuales: un estudio de caso en la formación de profesores de física. Estudios pedagógicos Vol.43 no.3 Valdivia 2017 http://dx.doi.org/10. 4067/S0718-0705201700030001 versión On-line ISSN 0718-0705

(8) Jofré , G. (2009). Competencias profesionales de los docentes de enseñanza media de Chile, Una percepción desde los implicados. Tesis doctoral, Universidad Autónoma de Barcelona. https://www.tdx.cat/bitstream/ handle/10803/5064/gjja1de2.pdf

(9) Mejía, G. (2018). El uso de la plataforma educativa EDMODO como recurso para la gestión de actividades escolares. Revista Dilemas Contemporáneos: Educación, Política y Valores. Año: VI Número: 1 Artículo no.:24 Período: 1ro. http://www. dilemascontemporaneoseducacion/ politicayvalores.com/ 
(10) Muñoz, L. y Vargas, L. (2019). Edumat: herramienta web gamificada para la enseñanza de operaciones elementales. Campus Virtuales, 8(2), 9-17.

(11) Ocampo, A., Gómez, M. y Zambrano, D. (2016). Percepción del profesor sobre el uso del b-learning para fortalecer competencias laborales. Vol. 7, Núm. 2 / octubre 2015 - marzo 2016 / ISSN 2007-1094 revista de innovación educativa.

(12) Pérez, Á.y Hernández-Sánchez, A. (2020). Efectos del programa affective e-learning en el desarrollo de la Competencia Digital en estudiantes del Grado en Educación Primaria. Educatio Siglo XXI, 38(3 NovFeb), 129-150. https://doi.org/10. 6018/educatio.416431

(13) Sanchez, L. (2020). Impacto del aula virtual en el proceso de aprendizaje de los estudiantes de bachillerato general. Revista tecnológica-educativa docentes. Vol.9, Núm. 1(2020) IV CIVTAC. DOI: https://doi.org/10. 37843/rted.v9i1.105

(14) Srnicek, N. (2017). Platform Capitalism. Buenos Aires, Argentina: Caja Negra Editores.

(15) UNESCO (2018) https://es.unesco. org/themes/docentes

(16) Valencia, G. y Rodríguez, G. (2018). Una revisión bibliográfica de estudios empíricos de herramientas de la Web 2.0 para el aprendizaje colaborativo: wikis, blogs, redes sociales y repositorios multimedia
(17) Vázquez, E., López, E., Fernández, E. y Ballesteros, C. (2018). Los nuevos entornos virtuales de aprendizaje permanente (MOOC) y sus posibilidades educativas en ámbitos sociales y educativos, Píxel-Bit. Revista de Medios y Educación. $\mathrm{N}^{\mathrm{O}}$ 53 Julio 2018. ISSN: 1133-8482, eISSN: 2171-7966. doi: http://dx.doi. org/10.12795/pixelbit.2018.i53.12

(18) Vilora, H. y Hamburger, J. (2019). Uso de las herramientas comunicativas en los entornos virtuales de aprendizaje, Chasqui. Revista Latinoamericana de Comunicación N. ${ }^{\circ}$ 140, abril - julio 2019 (Sección Informe, pp. 367-384), ISSN 1390-1079 / e-ISSN 1390-924X

(19) Viñas M. (2017). La importancia del uso de plataformas educativas. Revista Letras No. 6, junio 2017. Centro de Investigación en Lectura y Escritura (CILE) ISSN: 2524-938X, Páginas: 157-169 